\title{
Numerical Investigation of the Equivalent Impedance of a Wire Grid Parallel to the Interface Between Two Media
}

\author{
Tove Larsen
}

\author{
Contribution From Laboratory of Electromagnetic Theory, Technical University, Copənhagen, Denmark
}

(Received December 1, 1960; revised August 8, 1961)

\begin{abstract}
Based on a formula derived by Wait, a numerical investigation of the equivalent impedance of a wire grid parallel to the plane interface between two homogeneous media (ground and air) has been carried out. The calculations, which are of special interest to ground wire system design, are carried out for the grid placed in the air as well as in the ground.
\end{abstract}

\section{Introduction}

Many authors have investigated the electromagnetic properties of plane wire grids. Among these, Wait, in particular, has considered the case of a wire grid placed parallel to the plane interface between two homogeneous media, a configuration which is of great interest in investigations of ground wire systems for antennas. It is the purpose of this paper to describe numerical computations which have been carried out on the basis of Wait's formulas for the above-mentioned case.

\section{Formulas Derived by Wait}

In three papers Wait $(1956,1957,1958)$ has treated the case of a plane wire grid placed parallel to the interface between two homogeneous media.

Wait shows that under certain circumstances the two media and the grid may be considered equivalent to a composite transmission line being shunted with a certain impedance defined as the equivalent grid impedance. This description is valid for (a) oblique incidence, when the electric vector is parallel to the wires, (b) normal incidence for any polarization, (c) perfectly reflecting interface, for any angle of incidence and polarization, and (d) oblique incidence, when the magnetic vector is perpendicular to the wires.

The system which will be investigated in this paper is shown in figure 1a. It is a system which is of interest for a simple radial ground wire system of a vertical monopole. The incident wave is polarized in the plane of incidence, and this plane is parallel to the wires; i.e., the case considered here corresponds to the case mentioned above under (d). The equivalent transmission-line description is therefore valid. The equivalent circuit is shown in figure $1 \mathrm{~b}, K_{1}$ and $K_{2}$ being the characteristic impedances, $\Gamma_{1}$ and $\Gamma_{2}$ the propagation constants of the two transmission lines, and $Z_{g}$ being the equivalent impedance of the grid.
The grid consists of infinitely many, ideritical, equidistant circular wires of infinite length, with the radius $a$ and with the distance $d$ between adajcent wires. The grid is placed in the distance $h$ from the interface. The material parameters of the wires are $\epsilon^{\prime}, \mu^{\prime}$ and $\sigma^{\prime}$, whereas the medium 1 has the parameters $\epsilon_{1}, \mu_{1}$ and $\sigma_{1}$ and the medium 2 the parameters $\epsilon_{2}, \mu_{2}$, and $\sigma_{2}$, where $\epsilon$ is the dielectric constant, $\mu$ the permeability, and $\sigma$ the specific conductivity. It is assumed that $\mu^{\prime}=\mu_{1}=\mu_{2}=4 \pi \cdot 10^{-7} H / m$; i.e. the permeability of free space. The angle of incidence of the primary field is called $\theta_{1}$. The corresponding refraction angle $\theta_{2}$ is obtained from Snell's law;

$$
\sin \theta_{2}=\frac{k_{1}}{k_{2}} \sin \theta_{1}
$$

where $k_{1}$ and $k_{2}$ are the specific propagation constants of the two media. Using as time factor $e^{-i \omega t}$ we define the specific propagation constant by

$$
k=\omega \sqrt{\mu \epsilon\left(1+i \frac{\sigma}{\omega \epsilon}\right)},
$$

where $\omega$ is the angular frequency. The characteristic impedance $\zeta$ for the two media is given by

$$
\zeta=\sqrt{\frac{\mu}{\epsilon\left(1+i \frac{\sigma}{\omega \epsilon}\right)}} .
$$

The wavelength $\lambda$ in the two media is defined by the equation

$$
\lambda=\frac{2 \pi}{k}
$$

and it is assumed that

$$
a \ll d \ll|\lambda| \text {. }
$$


Under the above assumptions the magnitudes occurring in the equivalent diagram in figure $1 \mathrm{~b}$ are expressed in the following way:

The characteristic impedances of the equivalent transmission lines are

$$
\begin{aligned}
& K_{2}=\zeta_{2} \cos \theta_{2}, \\
& K_{1}=\zeta_{1} \cos \theta_{1},
\end{aligned}
$$

and the corresponding specific propagation constants are given by

$$
\begin{aligned}
& \Gamma_{2}=-i k_{2} \cos \theta_{2}, \\
& \Gamma_{1}=-i k_{1} \cos \theta_{1} .
\end{aligned}
$$

The equivalent grid impedance is given by

$$
Z_{g}=d Z_{i}-\frac{i \omega \mu d \cos ^{2} \theta_{1}}{2 \pi}\left\{\ln \frac{d}{2 \pi a}-R^{\circ} T+\Delta\right\},
$$

where $Z_{i}$ is the internal impedance per unit length of the wires

$$
Z_{i}=\frac{1}{2 \pi a} \sqrt{\frac{\mu \omega}{2 \sigma^{\prime}}}(1-i)
$$

The coefficient $R^{\circ}$ is given by and

$$
\begin{aligned}
& N_{1}=\sqrt{m^{2}-\left(\frac{d \cos \theta_{1}}{\lambda_{1}}\right)^{2}}, \\
& N_{2}=\sqrt{m^{2}-\left(\frac{d \cos \theta_{2}}{\lambda_{2}}\right)^{2}} .
\end{aligned}
$$

The formulas are valid for media with arbitrary constants. The following considerations are mainly confined to the case of medium 1 being air and medium 2 being ground.

\section{Discussion of Formulas}

The above-mentioned formulas were derived by Wait for the case, where the grid is placed in the medium 1 (air) in front of (seen from the generator) the interface as shown in figure 1 . The equivalent impedance of the grid when placed in medium 2 (ground) may be derived from these formulas by replacing index 1 by 2 and index 2 by 1 . Numerical signs have been put around $h$ in the formulas as we will let positive values of $h$ correspond to a grid in the air, and negative values of $h$ to a buried grid. We thus obtain for the two equivalent impedances $Z_{g a}$ (grid placed in air) and $Z_{g g}$ (grid placed in ground)

$$
R^{0}=\frac{\left[1+\left(\frac{k_{1} \cos \theta_{1}}{k_{2} \cos \theta_{2}}\right)^{2}\right]\left[1-\left(\frac{\cos \theta_{1}}{\cos \theta_{2}}\right)^{2}\right]+\sin ^{2} \theta_{1}\left[1-\left(\frac{k_{1} \cos \theta_{1}}{k_{2} \cos \theta_{2}}\right)^{2}\right]^{2}}{\left[1+\left(\frac{k_{1} \cos \theta_{1}}{k_{2} \cos \theta_{2}}\right)^{2}\right]\left[1-\left(\frac{\cos \theta_{1}}{\cos \theta_{2}}\right)^{2}\right]-\sin ^{2} \theta_{1}\left[1+\left(\frac{k_{1} \cos \theta_{1}}{k_{2} \cos \theta_{2}}\right)^{2}\right]^{2}} .
$$

The coefficient $T$ is given by

$$
T=\ln \left(1-e^{-\frac{2 \pi}{d}(2 \mid h i+a)}\right),
$$

and $\Delta$, which is a correction term that is negligible for $d \ll|\lambda|$, is given by

$$
\Delta=\sum_{m=1}^{\infty}\left\{\frac{1+R_{m} e^{-\frac{4 \pi|h|}{d} N_{1}}}{N_{1}}-\frac{1+R^{\circ} e^{-\frac{4 \pi|h|}{d} m}}{m}\right\},
$$

where

$$
\begin{gathered}
Z_{g a}=d Z_{i}-\frac{i \omega \mu d \cos ^{2} \theta_{1}}{2 \pi}\left\{\ln \frac{d}{2 \pi a}-R_{a}^{0} T+\Delta_{a}\right\} \\
Z_{g g}=d Z_{i}-\frac{i \omega \mu d \cos ^{2} \theta_{2}}{2 \pi}\left\{\ln \frac{d}{2 \pi a}-R_{g}^{0} T+\Delta_{g}\right\},
\end{gathered}
$$

where $R^{\circ}$ and $\Delta$ have the subscripts corresponding to air and ground, respectively.

In order to discuss these formulas more thoroughly the variation of some of the terms $\left(R^{\circ}, R_{m}, T, \Delta\right)$ will at first be considered, whereafter some simplified expressions for the impedance valid in special parameter cases will be worked out.

$$
R_{m}=\frac{\left[N_{1}+N_{2}\left(\frac{k_{1} \cos \theta_{1}}{k_{2} \cos \theta_{2}}\right)^{2}\right]\left[N_{1}-N_{2}\left(\frac{\cos \theta_{1}}{\cos \theta_{2}}\right)^{2}\right]+m^{2} \sin ^{2} \theta_{1}\left[1-\left(\frac{k_{1} \cos \theta_{1}}{k_{2} \cos \theta_{2}}\right)^{2}\right]^{2}}{\left[N_{1}+N_{2}\left(\frac{k_{1} \cos \theta_{1}}{k_{2} \cos \theta_{2}}\right)^{2}\right]\left[N_{1}+N_{2}\left(\frac{\cos \theta_{1}}{\cos \theta_{2}}\right)^{2}\right]-m^{2} \sin ^{2} \theta_{1}\left[1-\left(\frac{k_{1} \cos \theta_{1}}{k_{2} \cos \theta_{2}}\right)^{2}\right]^{2}}
$$



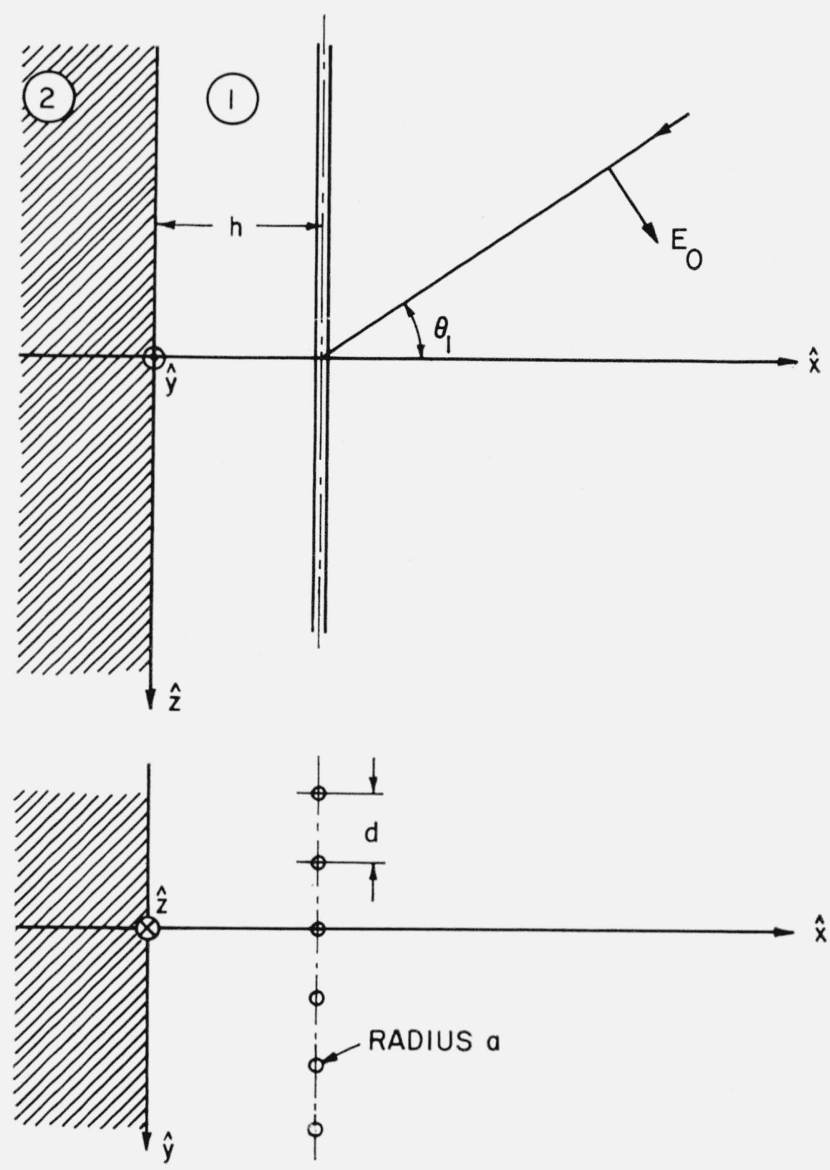

Figure 1a. Ground wire system.

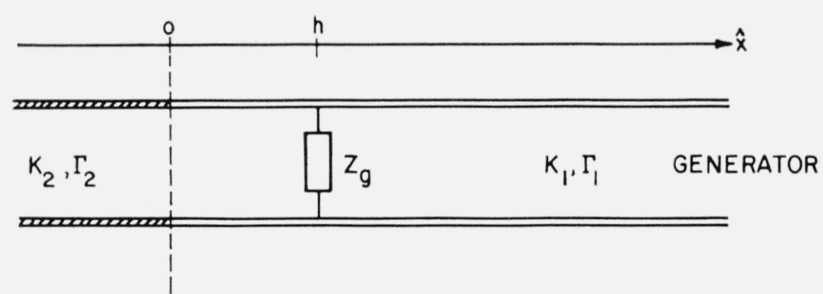

Figure 1b. The equivalent circuit of the ground wire system.

\subsection{Discussion of Single Terms}

For the sake of briefness we will introduce the following quantities

$$
\begin{gathered}
x=\sin ^{2} \theta_{1} \\
q=\left(\frac{k_{1}}{k_{2}}\right)^{2} \\
\alpha=\frac{N_{2}}{N_{1}} \\
\beta=\frac{m}{N_{1}} .
\end{gathered}
$$

\subsubsection{The Coefficient $\mathrm{R}^{\circ}$}

The expression (12) for the coefficient $R^{\circ}$ given by Wait may be further condensed. We find

and

$$
R_{a}^{0}=\frac{1-q}{1+q} \frac{x}{1-x}=\frac{k_{2}^{2}-k_{1}^{2}}{k_{2}^{2}+k_{1}^{2}} \operatorname{tg}^{2} \theta_{1}
$$

$$
R_{g}^{0}=-\frac{1-q}{1+q} \frac{x q}{1-x q}=\frac{k_{1}^{2}-k_{2}^{2}}{k_{1}^{2}+k_{2}^{2}}+g^{2} \theta_{2}
$$

From these expressions it is seen that for $\theta_{1}=0^{\circ}$ (perpendicular incidence)

$$
R_{a}^{\circ}=R_{g}^{\circ}=0 .
$$

When the ground is fairly well conducting $\left(\sigma_{2} \gg \omega \epsilon_{2}\right)$ the expressions for $R^{\circ}$ will become simple for all values of the angle of incidence. In this case we have

which shows that

$$
q \simeq-i \frac{\omega \epsilon_{1}}{\sigma_{2}}
$$

$$
|q| \ll 1 \text {, }
$$

as $\epsilon_{1} \leqq \epsilon_{2}$ when medium 1 is air.

We thus obtain

$$
\begin{aligned}
& R_{a}^{\circ} \cong \operatorname{tg}^{2} \theta_{1} \\
& R_{g}^{\circ} \cong-q x, \quad \text { i.e., }\left|R_{g}^{\circ}\right| \ll 1 .
\end{aligned}
$$

\subsubsection{The Coefficient $\mathrm{R}_{m}$}

Introducing the quantities (20 to 23 ) in the expression (15) for $R_{m}$ we find

$R_{m a}=\frac{[1-q x+\alpha q(1-x)][1-q x-\alpha(1-x)]+\beta^{2} x(1-q)^{2}}{[1-q x+\alpha q(1-x)][1-q x+\alpha(1-x)]-\beta^{2} x(1-q)^{2}}$

and

$$
\begin{aligned}
& R_{m g}= \\
& \quad \frac{-[1-q x+\alpha q(1-x)][1-q x-\alpha(1-x)]+\beta^{2} x(1-q)^{2}}{[1-q x+\alpha q(1-x)][1-q x+\alpha(1-x)]-\beta^{2} x(1-q)^{2}},
\end{aligned}
$$

which show, that $R_{m a}$ and $R_{m g}$ only differ by a change in sign in the first term of the numerators.

\subsubsection{The Coefficient $\mathrm{T}$}

The coefficient $T$, which is given by (13)

$$
T=\ln \left(1-e^{-\frac{2 \pi}{d}(2|h|+a)}\right),
$$

is seen to vary monotonically with $h$ between the limits given for $|h| \rightarrow \infty$ and for $h=0$ 
For $|h| \rightarrow \infty$ we have

$$
T \rightarrow \ln 1=0
$$

$$
\Delta_{a}(h \rightarrow \infty) \cong\left(\frac{d}{\lambda_{1}}\right)^{2} \frac{1-x}{2} \sum_{m=1}^{\infty} \frac{1}{m^{3}}
$$

and for $|h|=0$ we get

$$
\begin{aligned}
T \cong \ln \left(1-\left(1-\frac{2 \pi a}{d}\right)\right) & =-\ln \frac{d}{2 \pi a} \cdot \quad(31) \mid \\
\Delta_{a}(h=0) & \cong\left(\frac{d}{\lambda_{1}}\right)^{2} \frac{q^{3}\left(1-2 x+4 x^{2}\right)+q^{2}\left(3-12 x+4 x^{2}\right)+q(3-2 x)+1}{4 q(1+q)^{2}(1-x)} \sum_{m=1}^{\infty} \frac{1}{m^{3}} \\
\Delta_{g}(h=0) & \cong\left(\frac{d}{\lambda_{1}}\right)^{2} \frac{q^{3}\left(1-2 x+4 x^{2}\right)+q^{2}\left(3-12 x+4 x^{2}\right)+q(3-2 x)+1}{4 q(1+q)^{2}(1-q x)} \sum_{m=1}^{\infty} \frac{1}{m^{3}} .
\end{aligned}
$$

\subsubsection{The Correction Term $\Delta$}

In the following we shall find the first order approximations for the correction terms $\Delta_{a}$ and $\Delta_{g}$, which are supposed to be vanishingly small when $d \ll|\lambda|$. The terms are given by

$$
\begin{aligned}
& \Delta_{a}=\sum_{m=1}^{\infty} \frac{1}{m}\left\{\beta-1+\beta R_{m a} e^{-\frac{4 \pi|h|}{d} N_{1}}-R_{a}^{o} e^{-\frac{4 \pi|h|}{d} m}\right\} \\
& \Delta_{g}=\sum_{m=1}^{\infty} \frac{1}{m}\left\{\frac{\beta}{\alpha}-1+\frac{\beta}{\alpha} R_{m g} e^{-\frac{4 \pi|h|}{d} N_{2}}-R_{g}^{o} e^{-\frac{4 \pi|h|}{d} m}\right\} .
\end{aligned}
$$

We shall only calculate the sums for the extreme values $h=0$ and $|h| \rightarrow \infty$.

The quantities $\alpha$ and $\beta$ given by (22) and (23) are developed in powers of the quotient $\delta$ defined by

$$
\delta=\frac{1}{m^{2}}\left(\frac{d}{\lambda_{1}}\right)^{2} .
$$

Here and in what follows only the first order terms of $\delta$ are considered.

We thus obtain

$$
\alpha \cong 1-\delta \frac{1-q}{2 q}
$$

It is seen that the two last-mentioned sums are equal except for the factors $(1-x)$ and $(1-q x)$ in the denominators as they should be for $h=0$.

\subsection{Common Case}

In order to discuss the magnitude of the terms involved in the common expression for $Z_{g}$ we write (18) and (19) in the form

$$
\begin{aligned}
Z_{g a} & =d Z_{i} \\
& -\frac{i \omega \mu d}{2 \pi}\left\{\cos ^{2} \theta_{1} \ln \frac{d}{2 \pi a}-\cos ^{2} \theta_{1} R_{a}^{0} T+\cos ^{2} \theta_{1} \Delta_{a}\right\}
\end{aligned}
$$

$Z_{g g}=d Z_{i}$

$$
-\frac{i \omega \mu d}{2 \pi}\left\{\cos ^{2} \theta_{2} \ln \frac{d}{2 \pi a}-\cos ^{2} \theta_{2} R_{g}^{0} T+\cos ^{2} \theta_{2} \Delta_{g}\right\}
$$

where

$$
\begin{gathered}
\cos ^{2} \theta_{1} R_{a}^{0}=\frac{1-q}{1+q} x \\
\cos ^{2} \theta_{2} R_{g}^{0}=-\frac{1-q}{1+q} q x
\end{gathered}
$$

$$
\cos ^{2} \theta_{2} \Delta_{g}(h \rightarrow-\infty) \cong\left(\frac{d}{\lambda_{1}}\right)^{2} \frac{(1-q x)^{2}}{2 q} \sum_{m=1}^{\infty} \frac{1}{m^{3}}
$$

The numerical value of the factors $\cos ^{2} \theta_{1} R^{\circ}$ and $\cos ^{2} \theta_{2} R_{g}^{\circ}$ will always be less than unity, as the real part of $q$ never becomes negative. Therefore, as the most significant value of $T$ is $-\ln \frac{d}{2 \pi a}$, the greatest 
value of the second term in the bracket is $\ln \frac{d}{2 \pi a}$, the same as the greatest value of the first term in the brackets. The terms $\cos ^{2} \theta_{1} \Delta_{a}$ and $\cos ^{2} \theta_{2} \Delta_{g}$ are as will be shown numerically in the next section always very small as compared to the other terms in the brackets and may usually be neglected.

The term $d Z_{i}$ is usually much smaller than the other term in the expression for $Z_{g}$. Only for large positive $h(T \rightarrow 0)$ in the case of $\theta_{1}$ approaching $90^{\circ}$ $\left(\cos ^{2} \theta_{1} \ln \frac{d}{2 \pi a} \rightarrow 0\right) d Z_{i}$ becomes significant. Because of this, the phase angle of $Z_{g}$ is very near to $-90^{\circ}$ except for $\theta_{1}=90^{\circ}, \mathrm{h} \rightarrow+\infty$, where it is $-45^{\circ}$.

\subsection{Special Cases}

We shall now work out some simpler expressions for the equivalent impedances valid in special cases. The correction term $\Delta$ will be omitted.

\subsubsection{Grid in Interface, $h=0$}

In this case the two impedances $Z_{g a}$ and $Z_{g g}$ are equal and given by

$$
Z_{g a}=Z_{g g}=d Z_{i}-\frac{i \omega \mu d}{2 \pi} \ln \frac{d}{2 \pi a} \cos ^{2} \theta_{1,2}\left(1+R_{a, g}^{0}\right) .
$$

If the ground is assumed to be fairly well conducting, so that $R_{a}^{\circ} \cong t g^{2} \theta_{1}$ we get the following expression for the impedance.

$$
Z_{g}=d Z_{i}-\frac{i \omega \mu d}{2 \pi} \ln \frac{d}{2 \pi a}
$$

which shows, that $Z_{g}$ in this case is independent of the angle of incidence $\theta_{1}$ and of $\sigma_{2}$, provided only that $\sigma_{2} \gg \omega \epsilon_{2}$.

However, the same expression for $Z_{g}$ is obtained when the ground is poorly conducting, but only for $\theta_{1}=0^{\circ}$, as $R^{\circ}$ in this case is zero and $\cos ^{2} \theta_{1}=1$.

\subsubsection{Grid in Ground}

When $|h|$ becomes large we have that $T \rightarrow 0$ and consequently get

$$
Z_{g g}=d Z_{i}-\frac{i \omega \mu d}{2 \pi} \ln \frac{d}{2 \pi a} \cos ^{2} \theta_{2} .
$$

For the fairly well-conducting ground we have $\left|R_{g}^{\circ}\right| \ll 1$, and as the greatest value of $|T|$ is the same as the other term $\left(\ln \frac{d}{2 \pi a}\right)$ in the brackets we may neglect the term $R_{g}^{\circ} T$ and therefore get

$$
Z_{g g}=d Z_{i}-\frac{i \omega \mu d}{2 \pi} \ln \frac{d}{2 \pi a},
$$

the same value as was obtained above for $h=0$. There is consequently no variation of $Z_{g g}$ with $\theta_{1}, \mathrm{~h}$ or $\sigma_{2}$ when the grid is placed in a ground, which is fairly well-conducting.
Independent of the conductivity $\sigma_{2}$ we have $T_{g}^{\circ}=0$ for perpendicular incidence, $\theta_{1}=0$, and as $\cos ^{2} \theta_{2}=1$ in this case we again get the value

$$
Z_{g g}=d Z_{i}-\frac{i \omega \mu d}{2 \pi} \ln \frac{d}{2 \pi a} .
$$

\subsubsection{Grid in Air}

When $|h|$ becomes large we have $T \rightarrow 0$; we therefore obtain

$$
Z_{g a}=d Z_{i}-\frac{i \omega \mu d}{2 \pi} \ln \frac{d}{2 \pi a} \cos ^{2} \theta_{1},
$$

which is independent of the ground constants. From this expression it is seen, that for $\theta_{1} \rightarrow 90^{\circ}$ the equivalent grid impedance is exclusively determined by the internal impedance $Z_{i}$ and the distance between adjacent wires

$$
Z_{g a}=d Z_{i}
$$

As $T^{\circ}=0$ for perpendicular incidence, $\theta_{1}=0^{\circ}$, we again get the value

$$
Z_{g a}=d Z_{i}=-\frac{i \omega \mu d}{2 \pi} \ln \frac{d}{2 \pi a} .
$$

\subsubsection{Survey of Formulas for Special Cases}

A. The equivalent grid impedance is given by the expression

$$
Z_{g}=d Z_{i}-\frac{i \omega \mu d}{2 \pi} \quad \frac{d}{2 \pi a}
$$

in the following cases:

(a) $\theta_{1}=0^{\circ}, h$, and $\sigma_{2}$ arbitrary.

(b) Well-conducting ground, grid in ground (including $h=0), h, \theta_{1}$, and $\sigma_{2}$ arbitrary.

B. When the grid is placed in the ground-airinterface, $h=0$, of a poorly-conducting ground the impedance is

$$
Z_{g}=d Z_{i}-\frac{i \omega \mu d}{2 \pi} \ln \frac{d}{2 \pi a} \cos ^{2} \theta_{1,2}\left(1+R_{a, g}^{\circ}\right)
$$

C. When the grid is placed in the ground and $h \rightarrow-\infty$ the impedance is

$$
Z_{g}=d Z_{i}-\frac{i \omega \mu d}{2 \pi} \ln \frac{d}{2 \pi a} \cos ^{2} \theta_{2} .
$$

D. When the grid is placed in the air and $h \rightarrow \infty$ the impedance is

$$
Z_{g}=d Z_{i}-\frac{i \omega \mu d}{2 \pi} \ln \frac{d}{2 \pi a} \cos ^{2} \theta_{1} .
$$

E. When the grid is placed in the air, $\theta_{1}=90^{\circ}$ and $h \rightarrow \infty$ the impedance is

$$
Z_{g}=d Z_{i}
$$




\section{Numerical Computations}

In order to give a more instructive picture of the variation of the grid impedance numerical computations have been carried out for the following parameter values:

Frequency $f=200 \mathrm{kHz}$

Radius of the wires $a=0.001 \mathrm{~m}$

Specific conductivity of the wires $\sigma^{\prime}=5.8 \cdot 10^{7} \mathrm{~S} / \mathrm{m}$ (copper)

Medium 1 is air with

$\epsilon_{1}=8.854 \cdot 10^{-12} \mathrm{~F} / \mathrm{m}$

$\sigma_{1}=0 \mathrm{~S} / \mathrm{m}$.

Medium 2 is ground with

$\epsilon_{2}=10 \epsilon_{1}=8.854 \cdot 10^{-11} \mathrm{~F} / \mathrm{m}$

$\sigma_{2}=10^{-2} \mathrm{~S} / \mathrm{m}$ or $10^{-5} \mathrm{~S} / \mathrm{m}$ (Fairly well conducting and poorly conducting ground, respectively).

The distance $d$ between the wires varies from small values (determined by the condition $d \gg a$ ) up to $8 \mathrm{~m}$, and as the wavelength in air is $1,500 \mathrm{~m}$, the condition $\frac{d}{\lambda_{1}} \ll 1$ is fullfilled.

The coefficient $q=\left(\frac{k_{1}}{k_{2}}\right)^{2}$ will for the two sorts of ground be given by :

$$
q \cong\left\{\begin{array}{cc}
-i 0.0011 & \left(\sigma_{2}=10^{-2} \mathrm{~S} / \mathrm{m}\right) \\
0.1 & \left(\sigma_{2}=10^{-5} \mathrm{~S} / \mathrm{m}\right)
\end{array}\right.
$$

so we have

$$
\left[\frac{\delta}{|q|}\right]_{\max } \cong\left\{\begin{array}{l}
2.6 \cdot 10^{-2}\left(\sigma_{2}=10^{-2} \mathrm{~S} / \mathrm{m}\right) \\
2.9 \cdot 10^{-4}\left(\sigma_{2}=10^{-5} \mathrm{~S} / \mathrm{m}\right)
\end{array}\right.
$$

which shows that the condition (37) is fulfilled for both sorts of ground.

The correction term $\Delta \cos ^{2} \theta$, which is proportional to $d^{2}$ will be calculated for $d=8 m$, the greatest value of $d$ used in this investigation. As we have

$$
\sum_{m=1}^{\infty} \frac{1}{m^{3}}=1.202 \ldots
$$

we find

$$
\begin{aligned}
& h \quad \Delta \cos ^{2} \theta \quad \Delta \cos ^{2} \theta \\
& \left(\theta_{1}=0^{\circ}\right) \quad\left(\theta_{1}=90^{\circ}\right) \\
& \sigma_{2}=10^{-2} S / m\left\{\begin{array}{rrl}
+\infty & 1.7 \cdot 10^{-5} & 0 \\
0 & i 7.8 \cdot 10^{-3} & i 7.8 \cdot 10^{-3} \\
-\infty & i 1.6 \cdot 10^{-2} & i 1.6 \cdot 10^{-2}
\end{array}\right. \\
& \sigma_{2}=10^{-5} S / m\left\{\begin{array}{rll}
+\infty & 1.7 \cdot 10^{-5} & 0 \\
0 & 9.4 \cdot 10^{-5} & 8.1 \cdot 10^{-5} \\
-\infty & 1.7 \cdot 10^{-4} & 1.4 \cdot 10^{-4}
\end{array}\right.
\end{aligned}
$$

These quantities should in all cases except for $h=+\infty, \theta_{1}=90^{\circ}$, where all terms in the brackets are zero, be compared with a term of the order of magnitude $\ln \frac{d}{2 \pi a}$, which for $d=8 m$ equals 7.2 . This shows that in the present computations $\Delta \cos ^{2} \theta$ could be neglected in the case of the poorly conducting earth and that $\Delta \cos ^{2} \theta$ has a small influence in the case of the fairly well-conducting ground, however, the influence is so small that $\Delta \cos ^{2} \theta$ in most calculations may be neglected. This has been done in the computations described below.

Figures $2 \mathrm{a}$ and $\mathrm{b}$ and figures $3 \mathrm{a}$ and $\mathrm{b}$ show the variation of $Z_{g}$ with the distance $h$ of the grid from the ground surface with the angle of incidence $\theta_{1}$ as a parameter. Figure 2 applies for the fairly wellconducting ground $\left(\sigma_{2}=10^{-2} \mathrm{~S} / \mathrm{m}\right)$, whereas figure 3 applies for the rather poorly conducting ground $\left(\sigma_{2}=10^{-5} \mathrm{~S} / \mathrm{m}\right)$. In both cases curves have been given for the numerical value as well as for the phase of $Z_{g}$. The figures show that the grid impedance varies considerably with the angle of incidence when the grid is placed above the ground surface, whereas this variation is small when the grid is placed on or
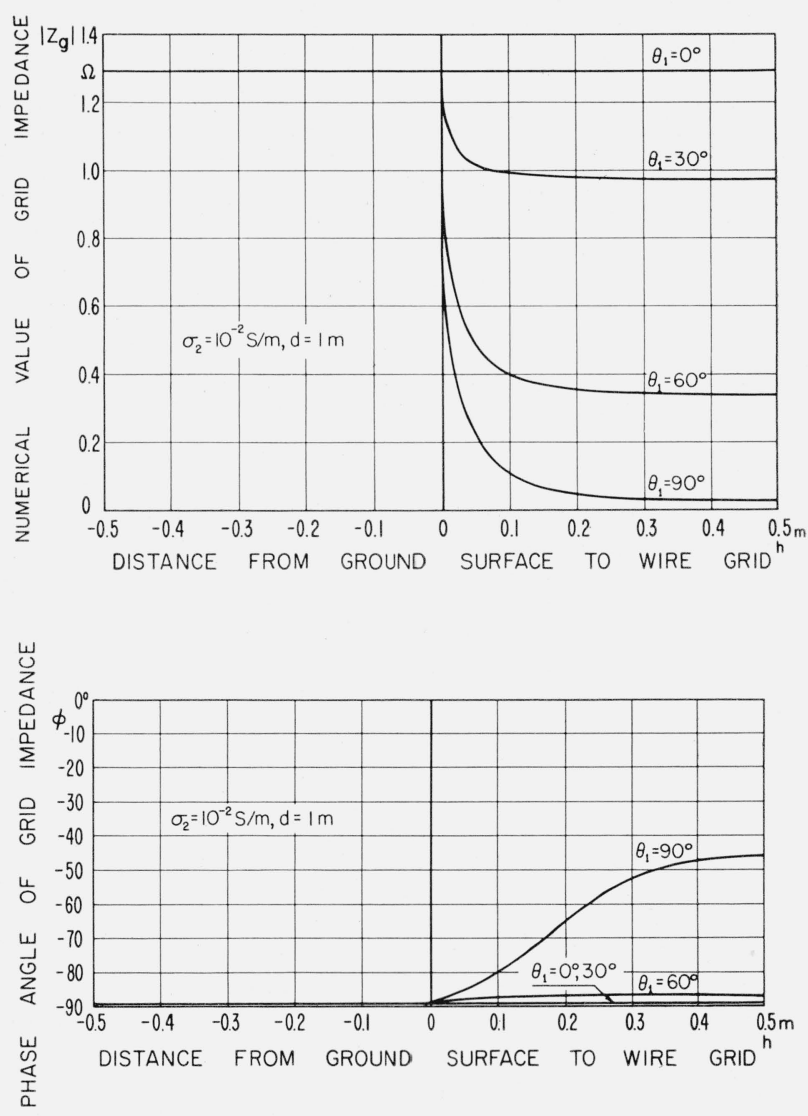

Figure 2. Equivalent grid impedance as a function of the distance from the wire grid to the ground-air interface (negative values of $\mathrm{h}$ correspond to a buried grid).

$$
\begin{gathered}
\text { Well-conducting ground } \sigma_{2}=10^{-2} S / m \text {. } \\
\text { a, Numerical value of impedance. } \\
\text { b, Phase angle of impedance. }
\end{gathered}
$$


below the surface; in the case where the ground is a good conductor there is practically no variation when the grid is placed in the ground, as found in the foregoing section.

The strong variation in the numerical value of the grid impedance in the case where the grid is placed in air is illustrated further by figures 4,5 , and 6 . In figure $4\left|Z_{g}\right|$ has been plotted as a function of $\theta_{1}$ with the specific conductivity of the ground $\sigma_{2}$ and the distance $d$ between the wires as parameters in the case where the wires are placed on the ground surface $(h=0)$, and when the presence of the ground surface is not taken into account $(h=\infty)$. Figure 5 shows corresponding curves for $d=1 \mathrm{~m}$ and for various values of $h$.

Finally figure 6 shows $\left|Z_{g}\right|$ as a function of the distance $d$ between the wires in some of the special cases mentioned in section 3.3.4., namely the cases A, B for $\theta_{1}=90^{\circ}$, and E. Curves of the phase of $Z_{g}$ corresponding to the three last mentioned sets of curves have been omitted since the variation of the phase angle in these cases is very small.

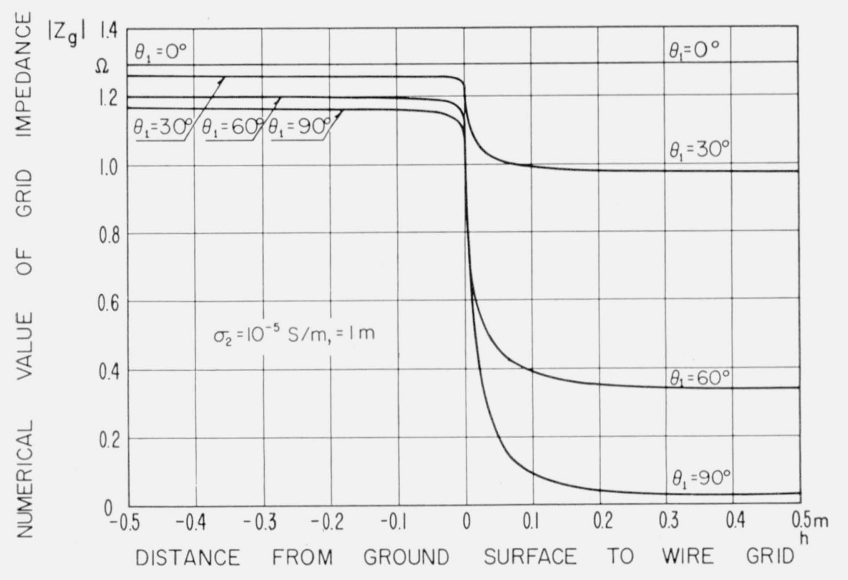

U

Figure 3. Equivalent grid impedance as a function of the distance from the wire grid to the ground-air interface (negative values of $\mathrm{h}$ correspond to a buried grid).

\footnotetext{
Poorly conducting ground, $\sigma_{2}=10^{-5} \mathrm{~S} / \mathrm{m}$. a, Numerical value of impedance.

b, Phase angle of impedance.
}

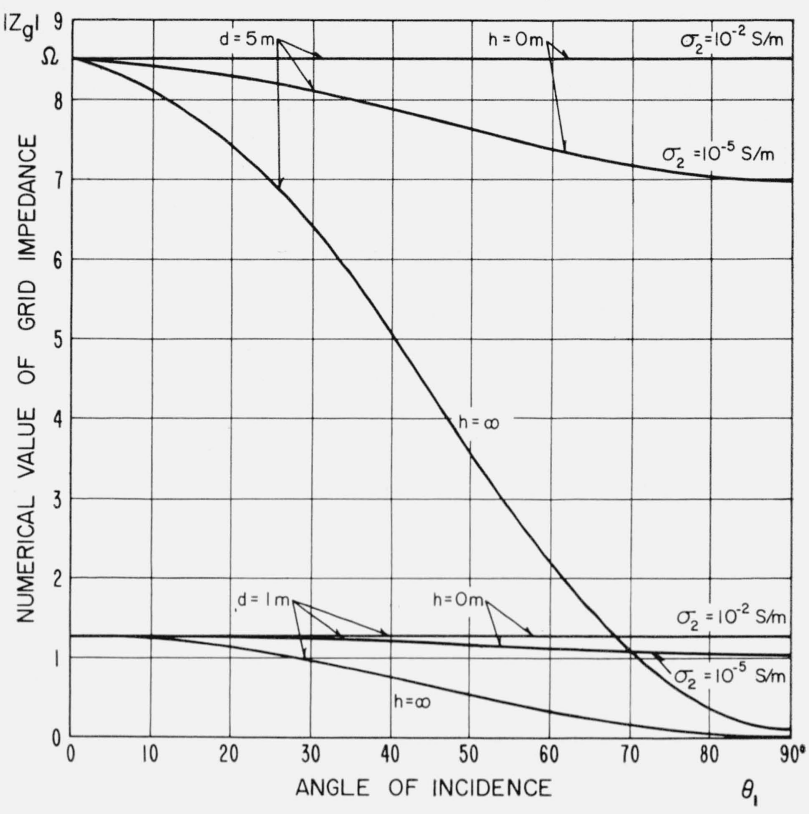

Figure 4. Numerical value of equivalent grid impedance as a function of the angle of incidence, when the grid is placed in the air.

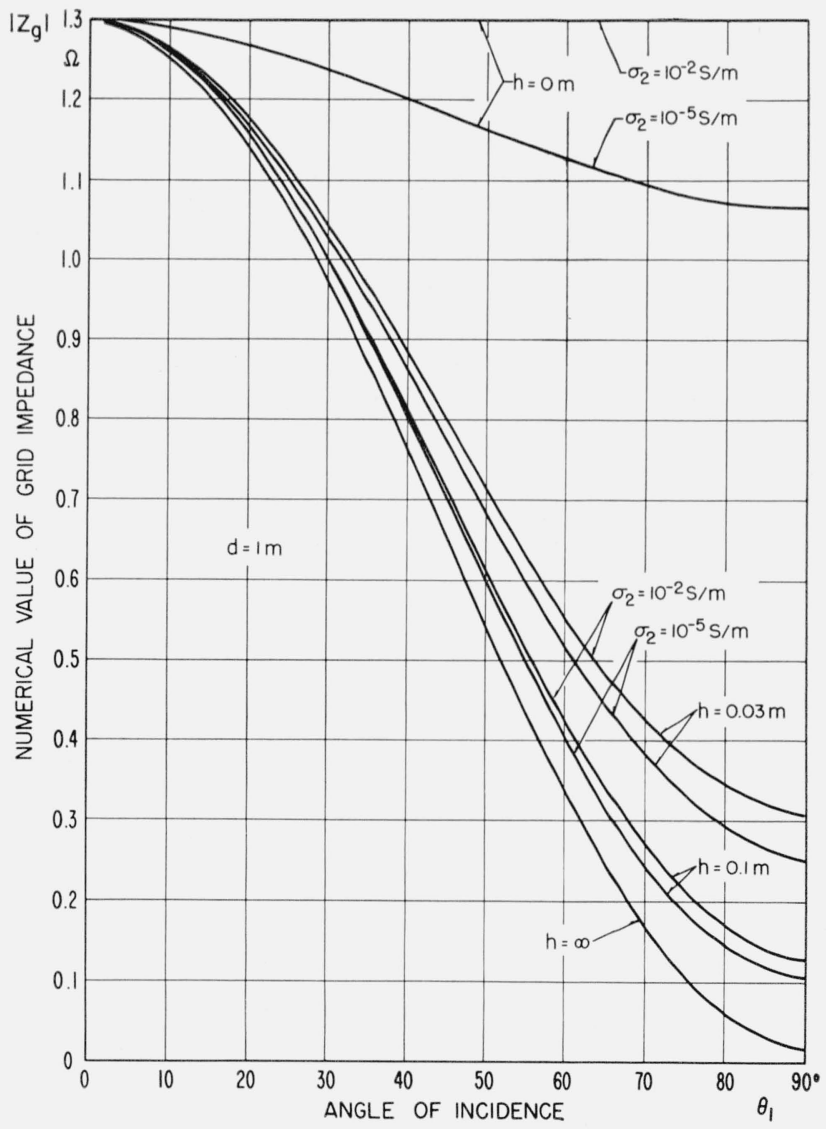

FIgure 5. Numerical value of the equivalent grid impedance as a function of the angle of incidence, when the grid is placed in the air. 


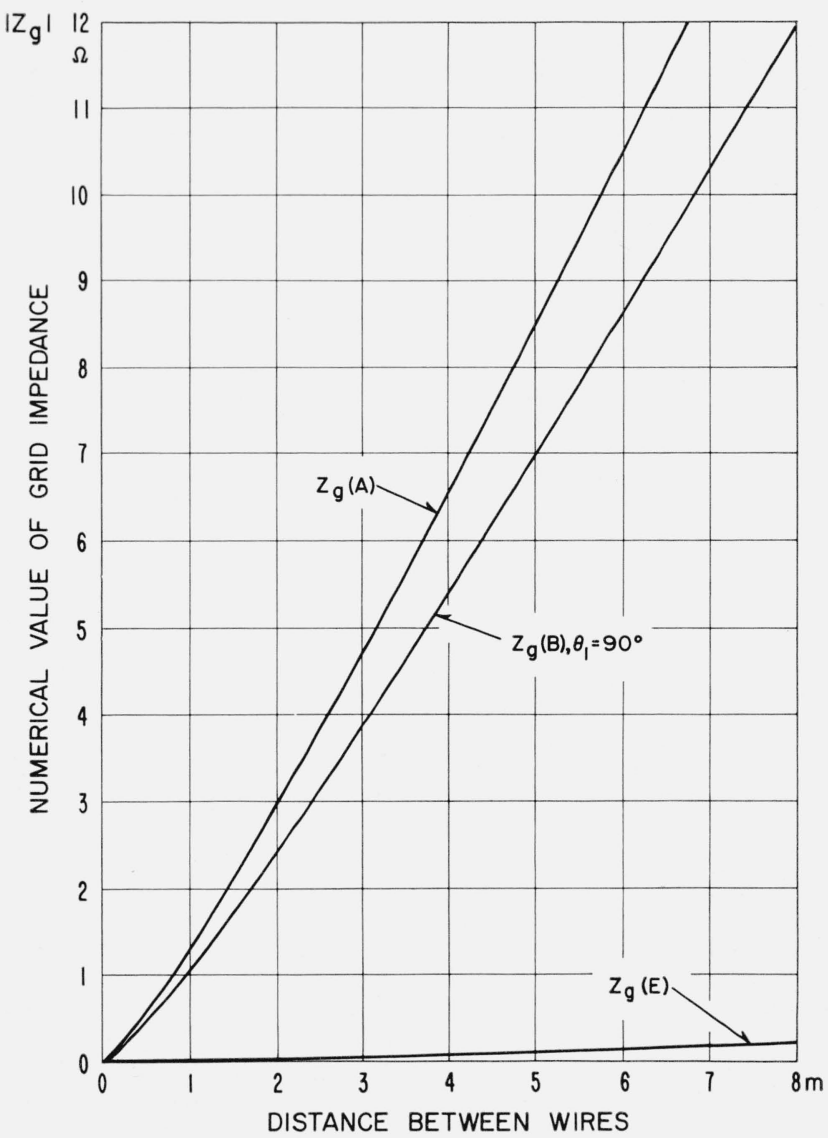

Figure 6. Numerical value of the equivalent grid impedance in some special cases as a function of the distance between adjacent wires.

$Z_{g}(\mathrm{~A})$ is valid for

(a) $\theta_{1}=0^{\circ}, h$ and $\sigma_{2}$ arbitrary

(b) Well-conducting ground, grid in ground includlng $h=0), h, \theta_{1}$, and $\sigma_{2}$ arbitrary.

$7_{g}(\mathrm{~B})$ is valid for $h=0, \sigma_{2}=10^{-5} \mathrm{~S} / \mathrm{m}$.

$7_{g}(\mathbf{E})$ is valid for a grid placed in the air, $h \rightarrow \infty, \theta_{1}=90^{\circ}$ $\sigma_{2}$ arbitrary.

\section{Conclusion}

The equivalent impedance of a wire grid placed parallel to the plane interface between air and ground has been investigated numerically, and curves have been plotted of this impedance as a function of the dimensions of the grid and the parameters of the ground.

The computations show that the approximation usually made in calculations regarding ground wire systems, namely to use the grid impedance corresponding to perpendicular incidence, is justified when the wire system is placed in the ground, but that considerable errors may occur when the grid is placed above the ground surface.

This investigation was carried out at the Laboratory of Electromagnetic Theory at the Technical University of Denmark by means of a grant from Den polytekniske Læreanstalts ingeniørvidenskabelige fond $\mathrm{og}$ G. A. Hagemanns Mindefond and by means of a support from the Air Force Cambridge Research Center, United States Air Force. Jesper Hansen assisted in preparing the final manuscript.

\section{References}

Wait, J. R., On the theory of reflection from a wire grid parallel to an interface between homogeneous media I, Appl. Sci. Research B6 259-275 (1956).

Wait, J. R., The impedance of a wire grid parallel to a dielectric interface, Trans. I.R.E. MTT-5 99-102 (1957).

Wait, J. R., On the theory of reflection from a wire grid parallel to an interface between homogeneous media II, Appl. Sci. Research B7 355-360 (1958).

(Paper 66D1-169) 